Research Article

\title{
An Effective Quantum Genetic Algorithm Based on Drama Resource Mining Using Wireless Sensing Technology
}

\author{
Rui Dong \\ Chinese Opera Academy, Shandong University of Arts, Jinan, Shandong 250300, China \\ Correspondence should be addressed to Rui Dong; z00601@sdca.edu.cn
}

Received 30 April 2021; Revised 7 June 2021; Accepted 10 June 2021; Published 25 June 2021

Academic Editor: Muhammad Usman

Copyright (c) 2021 Rui Dong. This is an open access article distributed under the Creative Commons Attribution License, which permits unrestricted use, distribution, and reproduction in any medium, provided the original work is properly cited.

\begin{abstract}
Wireless sensor technology has penetrated various domains of today's life and plays a vital role in the advanced technology. Numerous researchers have combined this outstanding technology with other fields such as resource mining, industry, healthcare, automobile system, gaming industry, and dramas. However, in traditional resource mining, long mining time leads to incomplete mining results along with low accuracy. In order to improve the effect of resource mining, we have proposed an effective Quantum Generic Algorithm (QGA) based on drama resource mining by using wireless sensor technology. In our proposed scheme, we have combined the RFID technology of wireless sensor with wireless network protocol stack for the purpose of collecting drama resources on the network platform. We have classified the drama resources on the network platform by using QGA based on the results of resources collection. Additionally, we have mined the semantic association features of frequent patterns of the drama resources on the platform and combined with the fuzzy attribute feature detection method. The experimental results show that this method is superior to the traditional methods in terms of resource mining time, mining results' comprehensiveness, and mining results' accuracy, which shows that this method has practical application value.
\end{abstract}

\section{Introduction}

Drama is derived from a Greek word that means "to do" or "to act." It is essentially a story that is acted out. And every play, whether serious or comedic, ancient or modern, conveys its tale through people in scenarios that are based on real life. Additionally, another key objective of drama is to generate a situation wherein the inferences, feelings, knowledge, and skills in education are liberated. In this connection, opera is a type of drama wherein music plays a central role and vocalists perform dramatic roles, but it differs from musical theater [1]. Opera is the product of combining Chinese opera art and TV media. The development and maturity of TV drama is the combination of TV as a modern means of communication and drama as a cultural resource $[2,3]$. Drama represents the integration of resources needed to develop drama and the implementation of localization and characteristic strategy of TV drama $[4,5]$. How to effectively obtain drama resources in the network platform has become a significant problem to be solved [6].
Many authors combined drama with today's modern technologies such as big data, wireless sensor technology, and artificial intelligence to facilitate human from low cost, high speed, and low data consumption.

In this regard, the authors in [7] proposed a data mining method based on FP growth algorithm to collect and extract features of power consumption information data of smart watt hour meter in operation, analyze abnormal power consumption data, apply machine learning method to learn eigenvalues, and deduce judgment threshold of power consumption abnormality; the association rule Data Mining method is used to fuse the results of independent detection, so as to realize the data mining of electricity theft. The experimental results show that this method can mine the abnormal data of power consumption in different periods, but it has the problem of long mining time. While in [8], the authors presented a data mining method for dam safety monitoring based on FP growth. After pruning the preprocessed monitoring data, priority tree is generated to mine frequent items. This method not only has the characteristics of fast mining 
speed and concise results, but also can compare single factor or analyze the relationship between multiple factor coupling and target variables, which provides a good idea for dam safety monitoring data mining. However, the main limitation of their work is that the results obtained by this method are not comprehensive, and there is a problem of missing data. Similarly, the authors of [9] planned a data mining method combining genetic algorithm and association rules is proposed. In their proposed work, firstly, GA crossover operator and mutation operator are improved adaptively, so that they can adjust adaptively according to the fitness value of function in the iterative process. Secondly, the improved adaptive GA is integrated into the association rules, making full use of GA's good global search ability to improve the efficiency of mining association rules with massive data. Thirdly, in order to avoid useless rules and reduce the existence of irrelevance, the intimacy degree is integrated to improve the reliability of association rules. Finally, on Hadoop big data platform, the optimized algorithm is verified by analyzing traffic data. The results show that the algorithm has the advantage of fast convergence speed, but the accuracy of data mining results is not high. In [10], a big data mining method for ocean going ship operation monitoring based on association rules is proposed. Firstly, the monitoring data source is obtained, and the data is stored in the database. Secondly, the ocean going ship operation monitoring data is preprocessed, so as to generate the ship operation monitoring big data mining model and complete the operation monitoring big data mining. The experimental results show that this method can ensure the accuracy of data mining, but it also has the problem of incomplete data mining results. All these works are facing limitations that need to be overcome.

Inspired from the current uprising of wireless sensing technology in various fields, specifically in the field of dramas, this study aims to develop a network platform wireless sensing technology for drama resource mining. The traditional methods are facing numerous problems regarding dramas such as long time mining, incomplete mining results, and low mining accuracy, so, in order to solve these problems, our proposed system provides efficient mining time and comprehensiveness of resource mining results with high accuracy by using Quantum Genetic Algorithm. In our proposed work, we have first designed a network platform to collect the drama resources; after that, we have acquired drama resource on the network platform based on wireless sensing technology by explaining its circuit diagram. We have also investigated Quantum Generic Algorithm based on our proposed drama resource mining and realized it.

The remainder of the paper consists of the following sections. We provide a list of related works in Section 2. In Section 3, we discuss our work strategy. The experimental attempt is discussed in Section 4. Finally, in Section 5, the study work's conclusion is presented.

\section{Related Work}

Drama is significant to a variety of academic areas, including cultural heritage transmission and multimedia repository classification and search. Tale ontologies [11-14] were proposed with two main purposes in mind: to identify story kinds and to provide an underlying model for narrative annotation. The authors used OWL to design several graphic kinds in [15]. The system employs the drama to execute casebased reasoning: provided a story plan, the system searches the drama for a plot that is comparable, calculating the semantic similarity of the provided plot to the plots recorded in the drama. In a similar vein, [16] utilizes automatic classifiers to categorize plot kinds, while the opiate system [17] creates and populates story worlds using a Proppian model of tale. A computational approach is used in [18] to create new stories in the manner of Russian fairy stories using the formal model. Several authors have questioned the extension of Propp's concept as a general story model in recent times, particularly in regard to digital media $[13,19]$.

One of the primary obstacles in the research on drama resource mining is resolving discrepancies between media kinds and genres. In $[20,21]$, the authors present the OntoMedia drama, a medium-independent paradigm that may be used in a variety of projects to document the narrative content of various media objects, spanning from written literature to comics and television drama. The logical notion of procedures, as used in SUMO, is used to reason about stories and produce storylines in [1]. Although not directly applicable to narrative frameworks, this method demonstrates the importance of proper action representation for tale characterization and annotation. Many initiatives have looked into the use of ontologies in online access to cultural material throughout the last decades. Computation ontologies, as discussed by [22], are particularly well suited to encoding conceptual models for access to digital resources and structuring the interaction between the archive and its users. The cultural Sampo initiative [23] makes a groundbreaking addition to the use of ontologies for culture and heritage accessibility. This project includes a set of domain ontologies that serve as a backdrop for exploring cultural items and monitoring their underlying relationships [24]. The system permits study of artifacts depending on their relationships with a reference tale at the narrative level; however, the story depiction is only functional for access to cultural items and is not meant as a standalone account of the narrative domain.

The authors in [25] advance the wireless sensor network coverage model by studying the operational features of the wireless sensor network. Though, it has better computational difficulty because it presents the optimization relations into the Particular Swarm Optimization (PSO). In [26], the authors planned a 2-phase system for gaining the finest energy provision technique by dealing with the game equilibrium of the design. Additionally, based on a connection model, in [27], the authors have presented a method of node optimization coverage for passive checking scheme of 3D-WSN. Currently, Quantum Optimization Algorithms have been progressively used to advance the network effectiveness of WSNs. For the purpose of improving the accuracy of positioning, the authors in [11] proposed a Positioning Algorithm based on quantum particle swarm optimization by using the parallelism of quantum computing. Though, the quality of the solution cannot be 
effectively improved by simply using Quantum Optimization Algorithm; therefore, there is a need to combine it with other techniques to further optimize the search capabilities of the algorithm. In $[4,5]$, the authors stated that drama represents the integration of resources needed to develop drama and the implementation of localization and characteristic strategy of TV drama, while in [6], the authors explained how to effectively obtain drama resources in the network platform has become a significant problem to be solved. Many authors combined drama with today's technologies, that is, big data, wireless sensor technology, and artificial intelligence, to facilitate human from low cost, high speed, and low data consumption [28].

\section{Proposed Work}

In this section, we discuss our proposed network platform for drama resource along with acquisition of drama resources on the network platform based on wireless sensor technology; after that, we will discuss Quantum Genetic Algorithm based on our proposed scheme, and at the end of this section, we will combine drama resources with the fuzzy attribute feature detection method for realization of the our proposed system [29].

\subsection{Network Platform Drama Resource Collection Platform.} In the process of drama resource mining on the network platform, drama resources are generally not indexed by search engines, and these high-quality drama resources cannot be directly obtained through search engines. This needs some mechanisms that enable search engines to obtain drama resources with high efficiency, thanks to drama resource mining, which has the ability to improve the coverage rate of drama resources by search engines [21]. Keeping in view of this, we have planned a drama resource collection platform that mainly includes page processing module, drama resource query interface recognition and classification module, and link construction and validity verification module. Furthermore, effective links and corresponding page contents in our proposed drama resources are found and used as resources to be searched by search engines. The drama resource collection platform mainly includes the following modules: page download and processing module, drama resource query interface recognition and classification module, link construction and validity verification module, and storage module. The overall schematic diagram is shown in Figure 1.

The following is a brief introduction to the functions implemented by each module in the drama resource collection platform of the network platform:

(1) Page download and processing module: the main task of this module is to obtain the page source code which enables page downloading and processing. Since the page source code contains a lot of impurity information, the page source code needs to be cleaned up and converted into a DOM tree for easy operation; otherwise, it will affect the efficiency of resource collection and processing [1].
(2) Drama resource query interface recognition and classification module: this is another important module of our proposed scheme. This module is connected with the users who seek drama resource. The main task of this module is to recognize the desired query and classify the drama resource query interface by field, removing irrelevant query interface.

(3) Link construction and validity verification module: this is another most important module of our proposed scheme, which is connected with drama resource query interface recognition and classification module. In this module, URL links are constructed mainly by querying keywords, querying drama resource keywords, at the same time, using more links to find more URL links, and verifying the validity of all URL links validity, filtering out the query results that cannot be obtained.

(4) Storage module: this is the last module of our proposed scheme that enable our system to save the verified valid URL link and its corresponding page information, so as to obtain the network platform drama resource collection result.

\subsection{Acquisition of Drama Resources on the Network Platform} Based on Wireless Sensor Technology. Because of the diversity of drama resources, it is not comprehensive to obtain the results of drama resources only through the network platform, so it is necessary to further collect the drama resources. As a new technology concept, the rapid development of wireless sensor technology has made it widely used in many fields such as consumer electronics, crop monitoring, livestock health monitoring, and medical services. In various factory environments, wireless local area network technology has been widely used as a communication information transmission tool between workers [30]. At the same time, radio frequency identification technology (RFID) as an electronic tag is also used in public transportation systems and personnel identification systems in the service industry widely used. The RFID system is explained in Figure 2.

The RFID tags in our proposed system are talented to identify every drama resource independently. This RFID is accomplished by analysis of numerous tags concurrently and instantly and can manage with strict and dirty environments. Furthermore, the RFID tags can also hold larger quantities of data, and data on tags can be read or simplified deprived of line of sight. These tag items are reusable and can also be automatically tracked out without the input of worker which eliminates the human errors, and they are not spoiled as simply like barcodes.

Our proposed scheme uses RFID technology in wireless sensor technology as a solution to collect drama resources on the network platform [31]. Among them, the wireless communication chip of the radio frequency transceiver module is TRY6831. In order to meet the low power consumption requirements of the node, the SQ series embedded microcontroller produced by TI is used as the main control module of the node. 


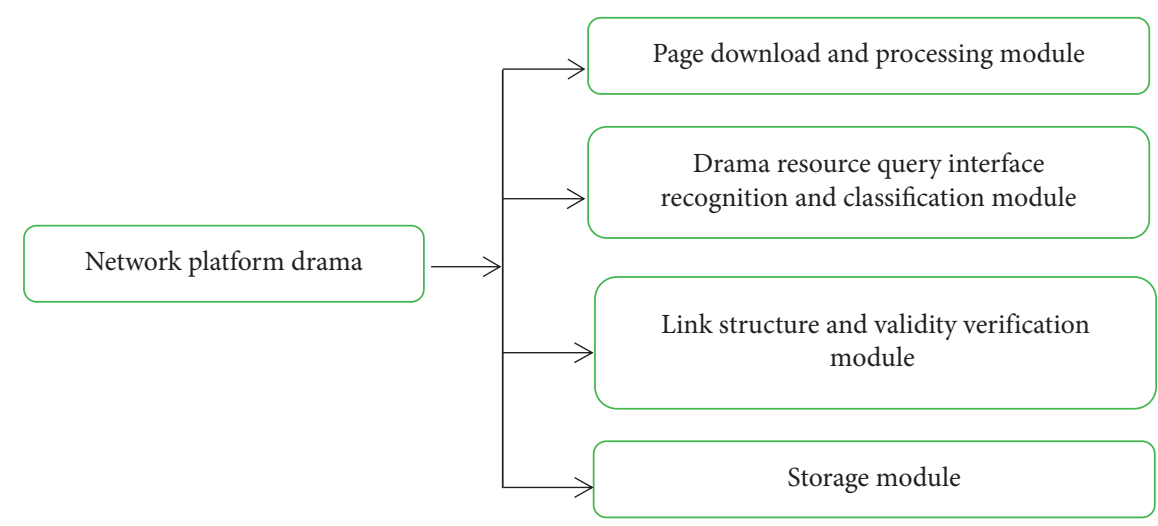

FIGURE 1: Schematic diagram of network platform drama resource collection platform.

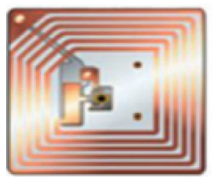

RFID tag

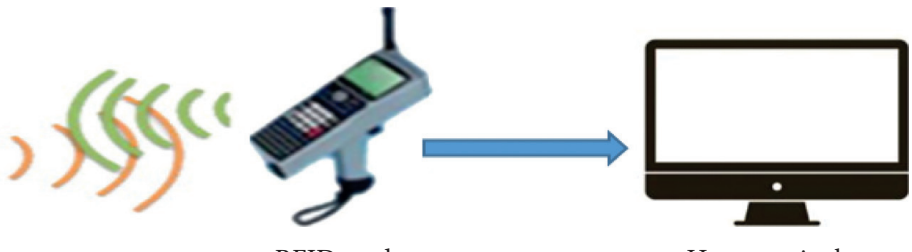

RFID reader

Host terminal

FIGURE 2: RFID system and its components.

The TXD end of the embedded single-chip microcomputer is connected to the RXD end of the sensor, the RXD end of the single-chip microcomputer is connected to the TXD end of the sensor, the power ground is the GND end, and the VCC end is connected to a $5 \mathrm{~V}$ power supply. The data resources are transferred to the embedded single-chip microcomputer to obtain the resource collection results. The circuit diagram of the network platform drama resource acquisition sensor is shown in Figure 3.

The resource collection nodes in Figure 3 are connected by a TRY6831 wireless communication chip. The chip has a transmission rate of $320 \mathrm{~kb} / \mathrm{s}$ and a transmission distance of $11.2 \mathrm{~m}$. It has the characteristics of high performance, low power consumption, and low cost.

The wireless sensor network protocol can be logically divided into two types: voice-oriented and data-oriented. In many wireless networks based on data transmission, small, low-cost, low-complexity wireless sensor networks are widely used. The wireless sensor network protocol essentially implements the connection of the entire protocol through the interface between the user and the protocol entity. For a specific layer user, it can call some services provided by the current layer protocol entity through service primitives. In the process, the current layer protocol entity will also call service primitives to return some status information to the user [32, 33]. The IEEE 802.15.4 and Zigbee alliance are committed to making low energy consumption, low-rate transmission, and low cost as important goals. The IEEE 802.15.4 standard and the Zigbee protocol specification have standardized the functions that should be implemented at each layer in the form of service primitives. The work of implementing the protocol is to implement the various primitives in the standard, aiming to provide a unified standard for the long-distance and low-speed interconnection between individuals and devices. IEEE 802.15.4 defines 13 PHY layer service primitives and 35 MAC layer service primitives.

(1) Physical layer (PHY): this indicates the physical layer which is mainly responsible for data modulation and demodulation, sending and receiving, directly operating the physical transmission medium (radio frequency) downwards, and providing services for the MAC layer upwards.

(2) Media access control (MAC): this layer is also called Data Link Layer, which is responsible for single-hop data communication between adjacent devices. It is also responsible for establishing synchronization with the network, supporting association and disassociation, and MAC security; it can provide a reliable connection between two devices.

(3) Network (NWK): this is the 3rd layer of our proposed Wireless Sensor Network Protocol Stack (Figure 4), which determines the mechanism used when devices are connected and disconnected from the network. This layer performs route discovery and route maintenance between devices. This layer also completes the discovery of neighboring devices within one-hop range and storage of related information, creates a new network along with it, and assigns network addresses for new networked devices [17]. 


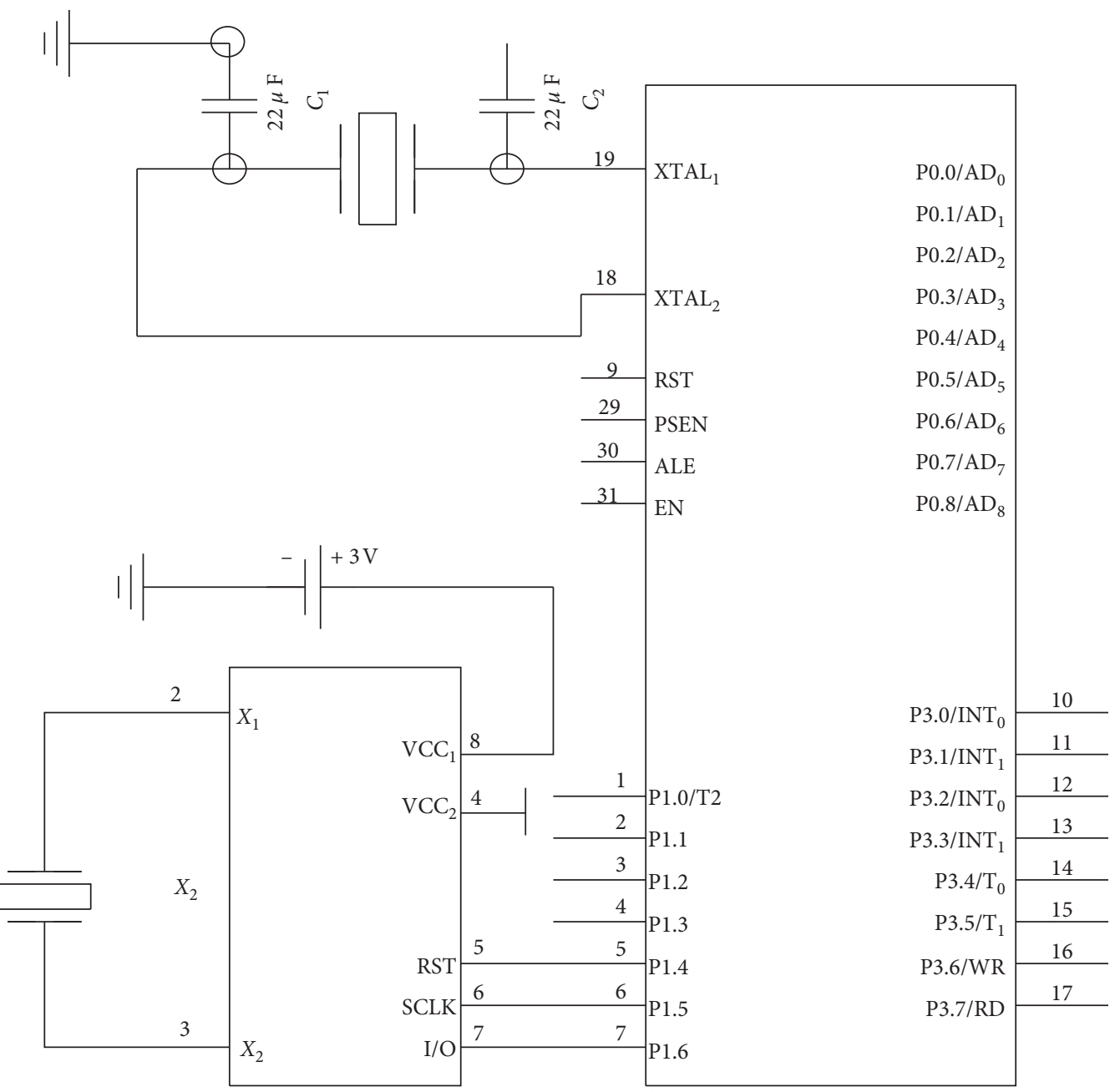

Figure 3: Network platform drama resource acquisition sensor circuit diagram.

(4) Application sublayer (APS): this is the 4th layer of the proposed Wireless Sensor Network Protocol Stack. This layer provides all endpoints services and connects to the device through the network layer and the security service provider layer and also provides services for data transmission, security, and binding. Therefore, it can adapt to different but compatible devices.

(5) Application layer (APL): this is the top most layer connected with the application software or user. This layer can configure and access network layer parameters through Zigbee Device Objects (ZDO) and provides them to application sublayer.

We have explained acquisition of drama resources and established wireless sensor network protocol stack; now, an algorithm is needed to classify the provided database and to mine the drama resources. In the next section, we will explain Quantum Genera Algorithm to achieve the desired goals.

3.3. Proposed Quantum Generic Algorithm for the Classification of Drama Resource. In this section, we discuss the classification algorithm (Quantum Generic Algorithm) for our proposed drama resource platform. As we know, classification is a form of data analysis that can be used to extract and describe important data categories. This analysis helps to understand the data better and comprehensively. There are many classification methods, such as the establishment of decision tree classifiers, naive Bayes classifiers, Bayesian belief networks, rule-based classifiers, and quantum genetic algorithms [34]. In the Quantum Genetic Algorithm, individuals are coded with the probability amplitude of qubits, the phase rotation of qubits based on quantum gates is used to realize individual evolution, and quantum NOT gates are used to realize individual mutation to increase the diversity of the population.

A qubit is a two-state quantum system that serves as an information storage unit. It is a unit vector defined in a twodimensional complex vector space [35]. This space consists of a pair of specific orthonormal basis $\{|0\rangle,|1\rangle\}$. Therefore, it can be in the superposition of two quantum states at the same time. It is defined as $|\beta\rangle=\varphi|0\rangle+\lambda|1\rangle$, where $\varphi$ and $\lambda$ are two complex numbers, representing the probability amplitude of the corresponding state, and satisfying the normalization condition $|\varphi|+|\lambda|=1$. A system containing $n$ qubits can represent $2^{n}$ states at the same time. When observing, the system will form a certain state. 


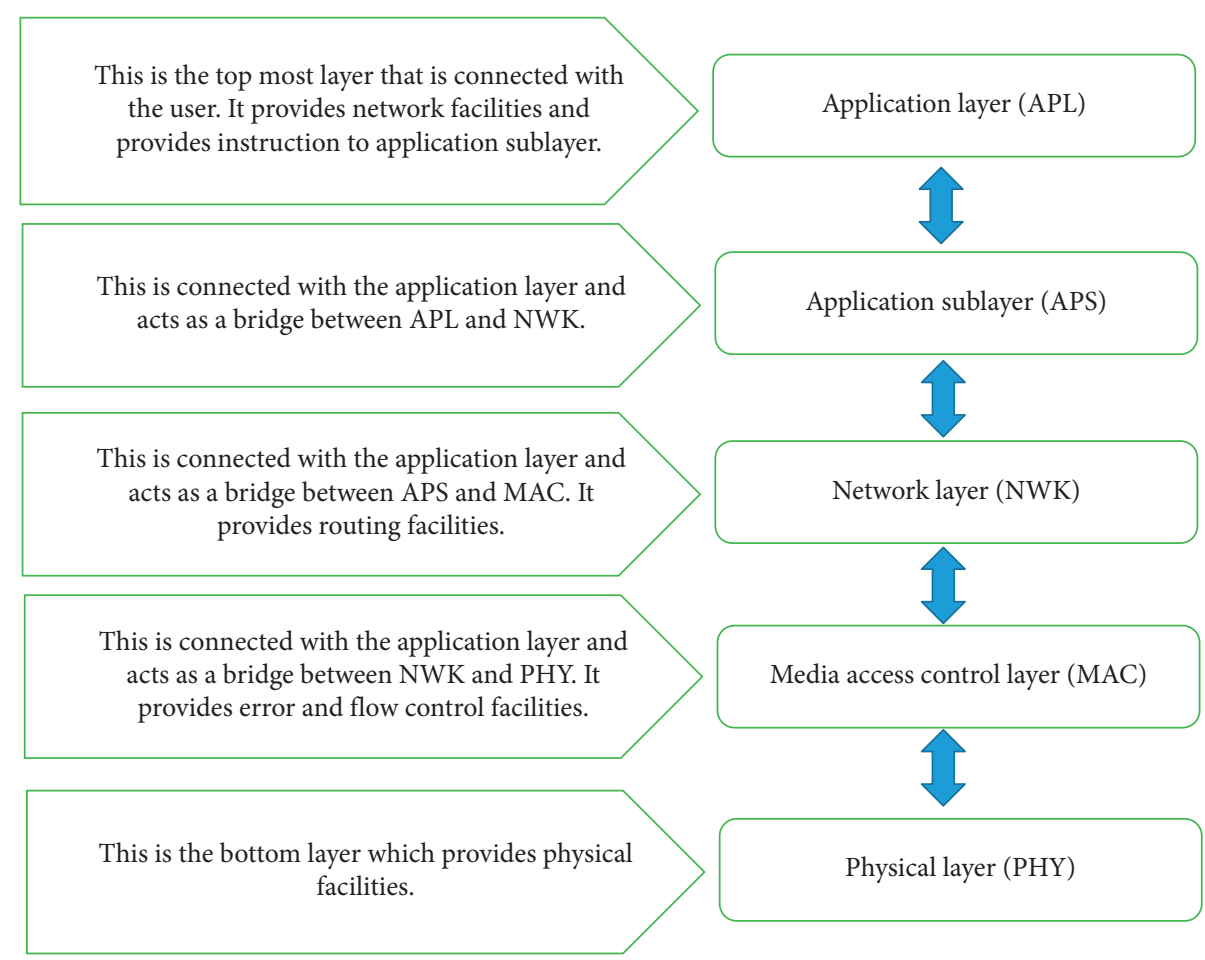

FIGURE 4: Wireless sensor network protocol stack.

There are many ways to encode chromosomes in traditional genetic algorithms: binary, decimal, symbolic encoding, etc. In the quantum genetic algorithm, an encoding method based on qubits is used. A qubit can be defined as

$$
\left[\begin{array}{l}
\varphi \\
\lambda
\end{array}\right]
$$

by its probability amplitude, and similarly, $k$ qubits can be defined as

$$
\left[\begin{array}{lll}
\varphi_{1} & \varphi_{2} & \varphi_{k} \\
\lambda_{1} & \lambda_{2} & \lambda_{k}
\end{array}\right]
$$

Among them, $\left|\varphi_{i}\right|+\left|\lambda_{i}\right|=1, i=1,2, \ldots, k$. This coding method makes the population have better diversity, and as $|\lambda|$ and $|\lambda|$ tend to 0 or 1 , the chromosome converges to a single state.

Our proposed Quantum Genetic Algorithm is based on the expression of quantum state vectors. It applies the probability amplitude representation of qubits to chromosome encoding, so that one chromosome can express the superposition of multiple state vectors, and uses quantum revolving gates to achieve chromosome update operations, the introduction of quantum mutation to overcome the premature phenomenon, and finally achieve the goal of optimization solution [36].

In the proposed Quantum Genetic Algorithm, the probability amplitude of a qubit can be expressed as $\left[\begin{array}{l}\varphi \\ \lambda\end{array}\right]$; then, the probability amplitude of $k$ qubits can be expressed by the following equation:

$$
\alpha_{k}=\left[\begin{array}{ccc}
\varphi_{1} \mid \varphi_{2} & \ldots & \varphi_{k} \\
\lambda_{1} \mid \lambda_{2} & \ldots & \lambda_{k}
\end{array}\right]
$$

Among them, the probability amplitude satisfies the normalization conditions given in the following equation:

$$
\left|\varphi_{i}\right|+\left|\lambda_{i}\right|=1 .
$$

Here, $i=1,2, \ldots, k$. If there is a quantum system with 3 bit quanta and three pairs of probability amplitudes, it can be expressed by the following equation:

$$
\left[\begin{array}{ccc}
\frac{1}{\sqrt{\varphi}} & 1 & \frac{1}{2} \\
\frac{1}{\sqrt{\varphi}} & 0 & \frac{\sqrt{\lambda}}{2}
\end{array}\right]
$$

Then, the state of the system can be described by the following equation:

$$
\frac{1}{2 \sqrt{\varphi}}|000\rangle+\frac{\sqrt{\varphi}}{2 \sqrt{\lambda}}|001\rangle+\frac{1}{2 \sqrt{\varphi}}|100\rangle+\frac{\sqrt{\varphi}}{2 \sqrt{\lambda}}|101\rangle .
$$

Therefore, the probability of the system appearing in states $|000\rangle,|001\rangle,|001\rangle$, and $|101\rangle$ is $1 / 8,3 / 8,1 / 8$, and $3 / 8$, respectively. Therefore, the three-bit quantum system described by the above equation can contain 4 states of information at the same time.

For the above equation, one chromosome can describe 4 states [37]. But in traditional evolutionary algorithms, 4 chromosomes are needed to describe 4 states, namely, (000), (001), (100), and (101). Populations described based on 
quantum chromosomes also have diversity. When $|\lambda|$ and $|\lambda|$ tend to 0 or 1 , the diversity will gradually disappear, and the quantum chromosome will converge to a certain state, which shows that the quantum chromosome has the ability to explore and develop at the same time.

Our proposed Quantum Genetic Algorithm is similar to the traditional Genetic Algorithm in that it is also a probabilistic search algorithm. Suppose a quantum population is given in the following equation:

$$
W(t)=\left\{w_{1}^{t}, w_{2}^{t}, \ldots, w_{n}^{t}\right\} .
$$

Here, $t$ represents the genetic algebra, while $w_{l}^{t}$ represents the $l$ chromosome of the $t$ generation, and the definition of $w_{l}^{t}$ is as shown in the following equation:

$$
w_{l}^{t}=\left[\begin{array}{ccc}
\varphi_{1}^{t} \mid \varphi_{2}^{t} & \ldots & \varphi_{m}^{t} \\
\lambda_{1}^{t} \mid \lambda_{2}^{t} & \ldots & \lambda_{m}^{t}
\end{array}\right] .
$$

Here, $m$ represents the qubit number, which is the length $L=1,2, \ldots, m$ of the chromosome. According to the above analysis, the process of our proposed platform drama resource classification algorithm based on quantum genetic algorithm is as follows.

3.4. Proposed Quantum Generic Algorithm. In this section, we present an effective quantum generic algorithm for our proposed drama resource mining.

$$
\begin{aligned}
& \text { Begin } \\
& \text { [Start Algorithm] } \\
& t=0 \text {; } \\
& \text { Initialize population } W(t) \\
& \text { [Initialization] } \\
& \text { Observe the state of } W(t) \text { to generate a binary solution } \\
& R(t) \\
& \text { Begin } \\
& t=t+1
\end{aligned}
$$

Observe the state of $W(t)$ and generate a binary solution $R(t)$

[Generate binary solution]

Calculate fitness function

[Calculate fitness]

Calculate the quantum gate update $W(t)$

[Calculate Quantum Gate]

Store the optimal solution

[Store solution]

End
3.5. Explanation of the Proposed Quantum Generic Algorithm. In this section, we explain the procedure of proposed Quantum Generic Algorithm. Here, the algorithm first initializes the population. When the population is initialized, all the probability amplitudes of $2^{m}$ of all chromosomes are initialized to $1 / \sqrt{2}$, which means that when the current number $t=0$, the linear superposition probability of each chromosome in all possible states is the same, which can be seen in the following equation:

$$
\left|\mu_{p}^{0}\right\rangle=\sum_{c=1}^{2^{n}} \frac{1}{\sqrt{2^{n}}} \mid h_{c} .
$$

Among them, $h_{c}$ represents the cth state, which is described by the binary string $\left(y_{1}, y_{2}, \ldots, y_{g}\right)$, $g=1,2, \ldots, m$.

Secondly, in the calculation process, the binary solution is set as follows: $K(t)$ is generated by observing the state of the population $W(t-1)$. Each solution is a binary string of length $L$, and its value is determined by the observation probability of the corresponding qubit. Then, calculate the fitness of each solution according to the obtained value to find the optimal solution.

In addition, in order to obtain a better chromosome, the binary solution

$$
K(t)
$$

is compared with the current optimal solution, and the population,

$$
W(t-1)
$$

is updated with an appropriate quantum gate $R(t)$. Specific quantum gates can be designed according to specific problems. The commonly used quantum revolving gate is shown in the following equation:

$$
R(t)=\left[\begin{array}{cc}
\cos \theta & -\sin \theta \\
\sin \theta & \cos \theta
\end{array}\right] \text {. }
$$

Here, $\theta$ represents the angle of rotation.

Finally, the optimal solution of the binary solution set $K(t)$ is selected. If the optimal solution is better than the optimal solution of the current platform drama resource classification, the optimal solution is used to replace the optimal solution of the current platform drama resource classification to realize the platform drama resource optimize classification.

3.6. Realization of the Mining of Drama Resources on the Network Platform. In this section, we discuss mining of proposed drama resource on the network platform. By mining the semantic association feature quantity of frequent patterns of platform drama resources, combined with the fuzzy attribute feature detection method, the network platform drama resources mining is realized. Combined with the autocorrelation feature detection method, the statistical analysis of frequent pattern mining of platform drama resources is carried out, and the fuzzy correlation fusion model of frequent pattern mining of platform drama 
resources is established; the feature segmentation model of platform drama resource frequent pattern data is established [37], which is expressed by the following equation:

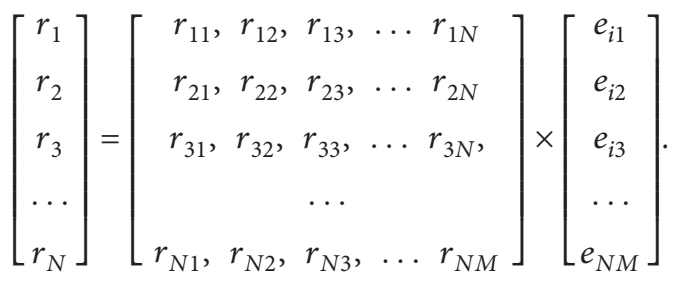

Among them, $r_{N M}$ represents the global weighted value of the frequent pattern data mining of platform drama resources at the Nth point, constructs the STARMA $(1,1)$ statistical analysis model of the frequent pattern data of graph data, and performs optimization control of the frequent pattern data mining of platform drama resources, expressed by the following equation:

$$
\rho_{k}=\left[\rho+(1-\rho) \frac{\eta}{W_{k}}\right]^{N} .
$$

Here, $\rho$ represents the fuzzy rule feature quantity of the frequent pattern mining of the platform drama resource data, using the statistical information analysis method, establish the platform drama resource frequent pattern data mining associated feature distribution set, and express it by the following equation:

$$
S_{h}=\frac{C_{\mathrm{ih}}+C_{\mathrm{oh}}-C_{\mathrm{io}}}{C_{\mathrm{io}}} \times \rho_{k} .
$$

Here, $C_{\text {ih }}$ represents the input space, $C_{\text {oh }}$ represents the output space, and $C_{\text {io }}$ represents the high-dimensional feature space. The calculation equations of the above three parameters are

$$
\begin{gathered}
C_{\text {ih }}=\frac{|C|}{|s|}, \\
C_{\text {oh }}=\frac{\mathrm{NB}}{|C|}, \\
C_{\text {io }}=\frac{\mathrm{NS}}{|S|} .
\end{gathered}
$$

Here, NB represents the closed frequent item set and NS represents the semantic segmentation domain.

Finally, the big data fusion method is used to perform pattern matching and information fusion clustering of frequent pattern mining of platform drama resources. At feature point $a$, the frequent pattern distribution set of platform drama resources is expressed as in the following equation:

$$
A=\left\{a_{1}, a_{2}, . ., a_{v}\right\}^{f}
$$

Here, $v$ represents the number of frequent pattern data of platform drama resources, and $f$ represents the weighting coefficient of frequent pattern mining of platform drama resources. Through the semantic dynamic feature segmentation method, the standard error coefficient of platform drama resource mining is obtained as in the following equation:

$$
X=x_{i}+A\left(x_{i \max }-x_{i \min }\right) .
$$

Here, $x_{i \max }$ represents the fuzzy constraint feature quantity of platform drama resource frequent pattern mining and optimization. Establish a storage module and an information query module for frequent pattern mining of platform drama resources, and establish a feature extraction and classification model for frequent pattern mining of platform drama resources to obtain the final mining output results given in the following equation:

$$
x_{k}=\sum_{i=1}^{N} w_{i} X^{k-1} .
$$

The result calculated by equation (19) is the output result of platform drama resource mining, thus completing the design of the network platform drama resource mining method based on wireless sensor technology.

\section{Simulation and Experimental Work}

In order to verify the effectiveness and comprehensiveness of the network platform drama resource mining method based on wireless sensor technology, simulation experiments are performed. Compared to the method used in $[7,8]$, we have used mining time, the comprehensiveness of the mining results, and the accuracy of the mining results as the experimental indicators.

4.1. Experimental Environment. We have carried out our experimental work for the proposed scheme, by using Linux Ubuntu 10.10 64-bit software, Intel Xeon E5606 4G memory $1 \mathrm{~T}$ hard disk, VIM editor and CodeBlock development tools, and $\mathrm{C}++$ as programming language.

4.2. Experimental Data. The experimental data comes from a local drama database, which includes three subdatabases: an index database, a data database, and a video database. The data sublibrary records information such as drama types, repertoires, characters, documents, pictures, and the video sublibrary records audio-visual data of the play. The index sublibrary provides indexes for the data sublibrary and the video sublibrary. Figure 5 shows the structure diagram of the database.

4.3. Experimental Results. In this section, first, we discuss the resource mining time, comprehensiveness of resource mining results, and accuracy of mining results. During our experimental work, we compare our scheme with the work of $[7,8]$.

4.3.1. Resource Mining Time. Comparing the mining time of different methods, the results are shown in Figure 5. In this figure, we have compared our proposed design with the 


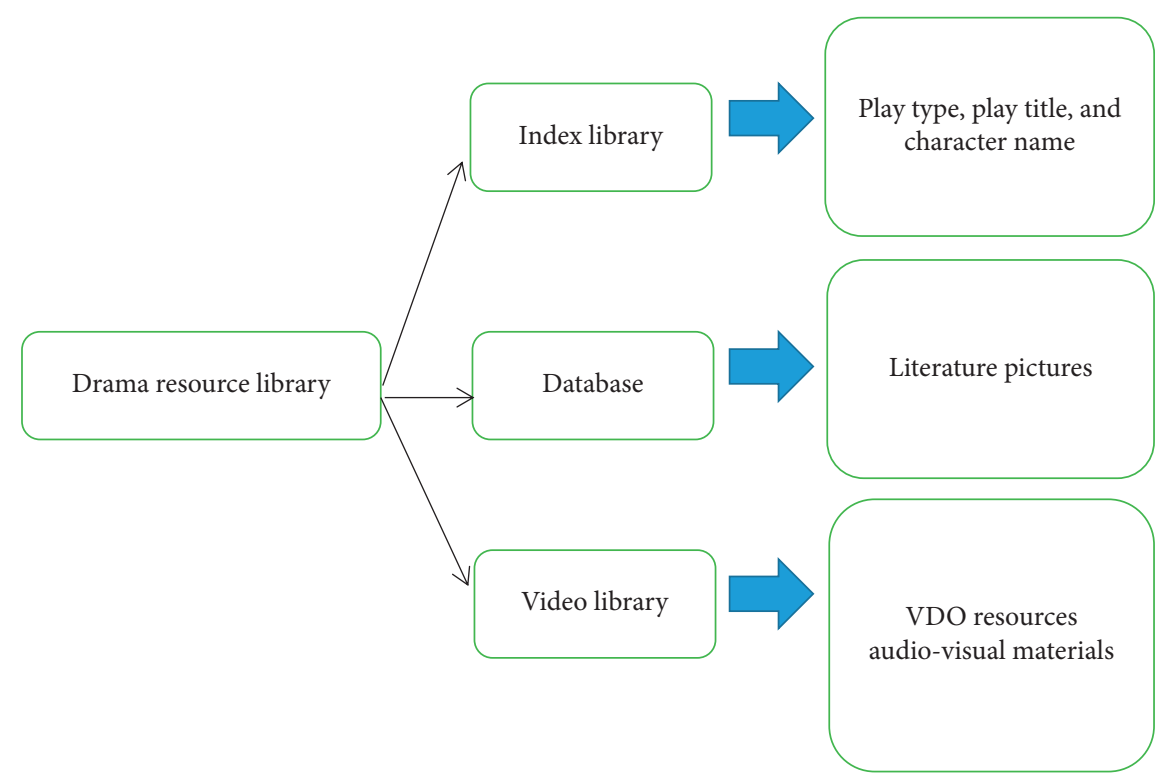

Figure 5: Structure diagram of drama database.

work of $[7,8]$. The rest will be explained in the coming section.

Analyzing Figure 6, it can be seen that the mining time consumed when mining resources using the method in this paper is significantly lower than that in reference [7] method and reference [8] method. The mining time of the method in this paper shows a continuous downward trend. When the number of the iterations is less than 6 times, the excavation time decreases significantly, and then the change trend of excavation time slows down, and the lowest value of excavation time is only $0.8 \mathrm{~s}$. By comparison, it can be seen that the mining time of this method is shorter, which shows that the resource mining efficiency of this method is higher.

4.3.2. Comprehensiveness of Resource Mining Results. Comparing the comprehensiveness of the resource mining results of different methods, the results are shown in Table 1 .

Analysis of the data in Table 1 shows that the method in this paper can mine up to 19 types of drama resources, and the lowest is 13 types, while reference [7] method and reference [8] method can mine fewer types of resources, far below the method of this research work. Among them, reference [7] method can only mine 9 drama resources at most, and reference [8] method can only mine 8 drama resources at most. Through comparison, it can be seen that the resource types obtained by mining method in this paper are more comprehensive, which shows that its application effect is better.

4.3.3. Accuracy of Mining Results. Comparing the accuracy of the mining results of different methods, the results are shown in Figure 6. In order to get the accurate results of mining, we have compared our proposed model with the work of $[7,8]$. The rest will be explained in the coming section.

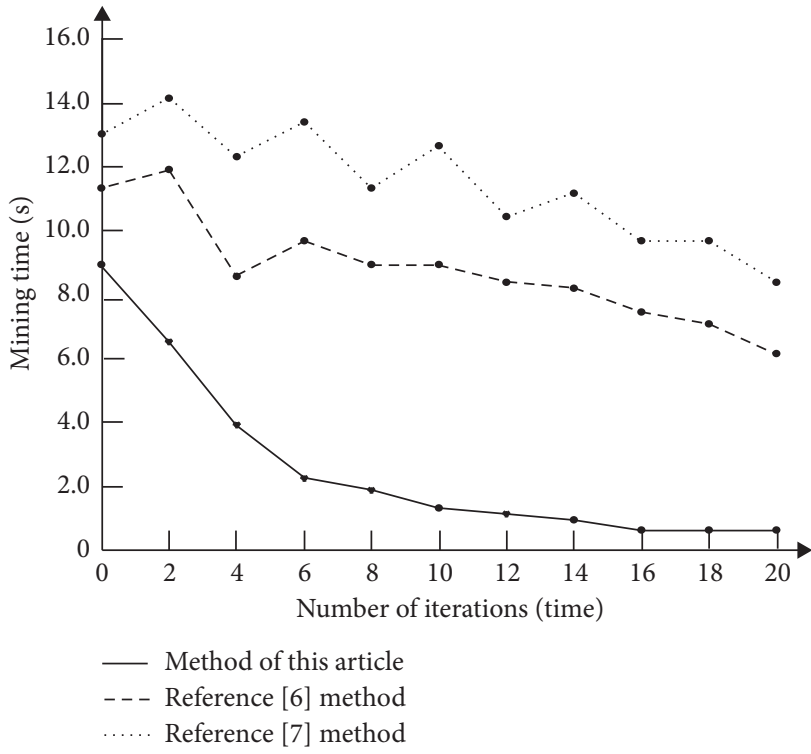

FIGURE 6: Comparison results of mining time.

According to Figure 7, the resource mining accuracy rate of this method is significantly higher than that of the traditional method. When the number of iterations is 5 , the mining accuracy rate of this method reaches $60 \%$, and the mining accuracy rate of the method in reference [7] method is $47 \%$; the mining accuracy rate of reference [8] method is $43 \%$. When the number of iterations is 14 times, the mining accuracy of the method in this paper reaches $82 \%$, the mining accuracy of the method in [7] is 56\%, and the mining accuracy of the method in [8] is $62 \%$. Through comparison, it can be seen that the mining results of the method in this paper are more accurate, indicating that the mining results are more reliable.

Comprehensive analysis of the above experimental results shows that the minimum mining time of this method is only $0.8 \mathrm{~s}$, which can mine up to 19 types of drama resources, and the 
TABLE 1: Comprehensive comparison of resource mining results of different methods.

\begin{tabular}{lccc}
\hline Number of iterations (time) & Resource type mining quality \\
[8] method & 7 & 15 \\
2 & 7 Method & 6 & 13 \\
4 & 9 & 8 & 14 \\
6 & 8 & 6 & 15 \\
8 & 7 & 6 & 16 \\
10 & 8 & 5 & 19 \\
12 & 6 & 7 & 15 \\
16 & 7 & 8 & 16 \\
\hline
\end{tabular}

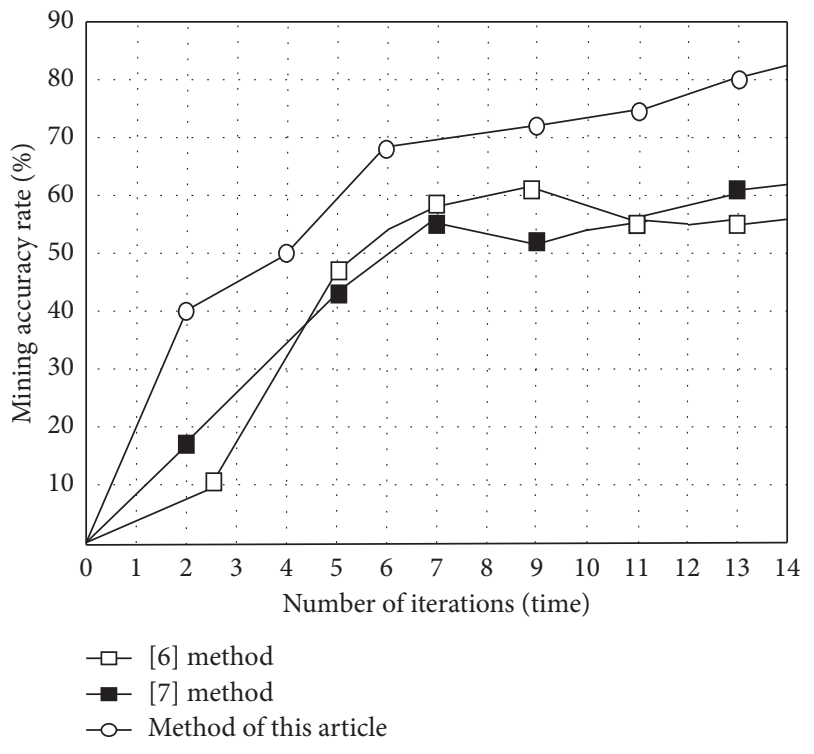

FIGURE 7: Comparison of accuracy of mining results of different methods.

mining accuracy rate reaches $82 \%$. This method has obvious advantages in mining time, mining comprehensiveness, and mining accuracy, which shows that the mining results of this method are more reliable and the mining efficiency is higher.

The network efficiency of the proposed QGA technique in target allocation is examined, and QGA provides a comparison with the Particle Swarm Optimization (PSO) and Simulated Annealing (SA) algorithms in wireless sensor technology for target allocation. Then, the efficiency of QGA is compared with different amounts of target points and number of sensors. The entire testing procedure is carried out on a computer using the same hardware and software.

Figure 8 shows how much iteration is necessary for SA, PSO, and our proposed QG algorithm to reach convergence under various simulated conditions. The range variation percentage judgment approach is used to determine convergence. The variation limit specified in this experiment is $1 \%$ to $2 \%$; that is, the algorithm can be regarded convergent if the rise percentage of network efficiency achieved by the algorithm is between $1 \%$ and $2 \%$. As a result, our suggested Quantum Generic Algorithm appears to have a better convergence performance.

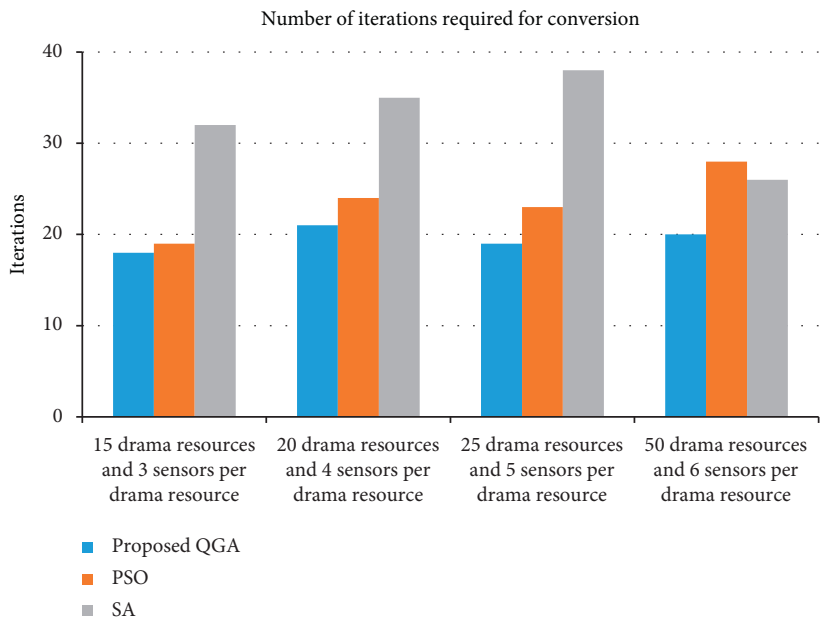

FIGURE 8: Conversion comparison of SA, PSO, and proposed QGA.

\section{Conclusion}

In conclusion, this paper puts forward an efficient Quantum Generic Algorithm (QGA) for drama resource mining based on wireless sensing technology. From the perspective of drama resource, this paper makes a mathematical model and then uses the proposed algorithm to solve the mathematical model. The results show that it is effective in solving the problem of resource mining time, comprehensiveness of mining results, and accuracy of the mining results into the sensor target allocation problem. The results prove the effectiveness of the QGA-based drama resource in optimizing network efficiency of the wireless sensing technology. By applying QGA, a more efficient drama resource mining scheme can be obtained when drama resource mining is carried out. Not only does the scheme obtain the maximum monitoring effect of the drama resource, but also it saves mining time and produces accurate results. Through the suitable deployment of sensors, limited resources can be used as much as possible. Future research should carefully consider other methods that can further improve the network efficiency of drama resource mining, such as combining routing optimization algorithms and clustering and machine learning techniques. 


\section{Data Availability}

The datasets used and/or analyzed during the current study are available from the corresponding author upon request.

\section{Conflicts of Interest}

The author declares that he has no conflicts of interest regarding the publication of this paper.

\section{References}

[1] E. Hyvonen, "Semantic portals for cultural heritage," in Handbook on Ontologies, pp. 757-778, Springer, Berlin, Germany, 2009.

[2] T. Itzel, M. Neubauer, M. Ebert, M. Evert, and A. Teufel, "Hepamine-a liver disease microarray database, visualization platform and data-mining resource," Scientific Reports, vol. 10, no. 1, p. 4760, 2020.

[3] N. W. Borsato, S. L. Martell, and J. D. Simpson, "Identifying stellar streams in Gaia DR2 with data mining techniques," Monthly Notices of the Royal Astronomical Society, vol. 492, no. 1, pp. 1370-1384, 2020.

[4] A. Hsrpd, P. T. Fukuda, P. Y. Tamaki et al., "Computerized data mining analysis of keywords as indicators of the concepts in AHA-BLS guideline updates," The American Journal of Emergency Medicine, vol. 38, no. 7, pp. 1436-1440, 2020.

[5] I. Parvez, J. Shen, I. Hassan, and N. Zhang, "Generation of hydro energy by using data mining algorithm for cascaded hydropower plant," Energies, vol. 14, no. 2, p. 298, 2021.

[6] I. Dumitracu-Bldu, D. D. Dumitracu, and G. Dobrot, "Predictive model for the factors influencing international project success: a data mining approach," Sustainability, vol. 13, no. 7, p. 3819, 2021.

[7] X. M. Duan, S. Wang, T. Zhao, and X. N. Ding, "Data mining method on abnormal electricity usage based on FP-growth algorithm," Application of Electronic Technique, vol. 46, no. 10, pp. 47-50, 2020

[8] N. N. Mao, H. Z. Su, and J. X. Gao, "Data mining method for dam safety monitoring based on FP-growth algorithm," Advances in Science and Technology of Water Resources, vol. 39, no. 5, pp. 78-82, 2019.

[9] H. Sun and C. J. Li, "Improvement of data mining method combining genetic algorithm and as-sociation rules," Journal of Data Acquisition \& Processing, vol. 34, no. 5, pp. 863-871, 2019.

[10] N. Liu and L. Wang, "Large data mining method for ocean ship operation monitoring based on association rules," Ship Science and Technology, vol. 41, no. 18, pp. 176-178, 2019.

[11] P. Gervas, B. Diaz-Agudo, F. Peinado, and R. Hervas, "Story plot generation based on CBR," Knowledge-Based Systems, vol. 18, no. 4, pp. 235-242, 2005.

[12] X. Yu, Y. Chu, F. Jiang, Y. Guo, and D. Gong, "SVMs classification based two-side cross domain collaborative filtering by inferring intrinsic user and item features," KnowledgeBased Systems, vol. 141, pp. 80-91, 2018.

[13] M. O. Jewell, K. F. Lawrence, M. M. Tuffield et al., "OntoMedia: an ontology for the representation of heterogeneous media," in Proceedings of the Multimedia Information Retrieval Workshop, ACM SIGIR, Singapore, January 2005.

[14] P. Gervas, "Propp's morphology of the folk tale as a grammar for generation," in Proceedings of the CMN Workshop on Computational Models of Narrative, A Satellite Workshop of
CogSci 2013: The 35th Meeting of the Cognitive Science Society, pp. 106-122, Germany, Berlin, August 2013.

[15] V. Propp, Morphology of the Folktale, University of Texas Press, Austin, TX, USA, 1968.

[16] K. Hartmann, S. Hartmann, and M. Feustel, "Motif definition and classification to structure non-linear plots and to control the narrative flow in interactive dramas," in Proceedings of the Virtual Storytelling. Using Virtual Reality Technologies for Storytelling, pp. 158-167, Strasbourg, France, November 2005.

[17] C. R. Fairclough, "Story games and the OPIATE system: using case-based planning for structuring plots with an expert story director agent and enacting them in a socially simulated game world," Doctoral Thesis, University of Dublin Trinity College, Dublin, Ireland, 2004.

[18] M. Cavazza and D. Pizzi, "Narratology for interactive storytelling: a critical introduction," in Proceedings of the Technologies for Interactive Digital Storytelling and Entertainment, pp. 72-83, Darmstadt, Germany, December 2006.

[19] Z. Tomaszewski and B. Kim, "The limitations of a proppbased approach to interactive drama," in Proceedings of the Intelligent Narrative Technologies: Papers from the AAAI Fall Symposium, pp. 167-173, Menlo Park, CA, USA, November 2007.

[20] K. F. Lawrence, "Crowdsourcing linked data from Shakespeare to Dr who," in Proceeding of the Web Science International Conference, New York, NY, USA, June 2011.

[21] J. Cua, E. Ong, R. Manurung, and A. Pease, "Representing story plans in SUMO," in Proceedings of the NAACL HLT 2010 Second Workshop on Computational Approaches to Linguistic Creativity, pp. 40-48, Association for Computational Linguistics, Los Angeles, CA, USA, June 2010.

[22] E. Hyvonen, E. Makela, T. Kauppinen et al., "CultureSampo: a national publication system of cultural heritage on the semantic web 2.0," in Proceedings of the 6th European Semantic Web Conference, pp. 851-856, Heraklion, Greece, May 2009.

[23] E. Hyvonen, E. Makela, T. Kauppinen et al., "CultureSampoFinnish culture on the semantic web 2.0. thematic perspectives for the end-user," in Proceedings of the Museums and the Web 2009, Indianapolis, IN, USA, April 2009.

[24] Y. Zhang, "Coverage optimization and simulation of wireless sensor networks based on particle swarm optimization," International Journal of Wireless Information Networks, vol. 27, no. 4, pp. 307-316, 2019.

[25] C. Gong, C. Guo, H. Xu, C. Zhou, and X. Yuan, "A joint optimization strategy of coverage planning and energy scheduling for wireless rechargeable sensor networks," Processes, vol. 8, no. 10, p. 1324, 2020.

[26] Z. Hao, N. Qu, X. Dang, and J. Hou, "Node optimization coverage method under link model in passive monitoring system of three-dimensional wireless sensor network," International Journal of Distributed Sensor Networks, vol. 15, no. 8, p. 155014771986987, 2019.

[27] W. L. Wu, X. B. Wen, H. X. Xu, L. M. Yuan, and Q. X. Meng, "Accurate range-free localization based on quantum particle swarm optimization in heterogeneous wireless sensor networks," KSII Transactions on Internet \& Information Systems, vol. 12, no. 3, pp. 1083-1097, 2018.

[28] M. Cataldi, R. Damiano, V. Lombardo, A. Pizzo, and D. Sergi, "Integrating commonsense knowledge into the semantic annotation of narrative media objects," in Proceedings of the AI * IA 2011: Artificial Intelligence Around Man and Beyond, pp. 312-323, Palermo, Italy, September 2011. 
[29] Y. Xu, J. Yang, and Z. Xie, "Training SVMs on a bound vectors set based on fisher projection," Frontiers of Computer Science, vol. 8, no. 5, pp. 793-806, 2014.

[30] J. Majrouhi Sardroud, "Influence of RFID technology on automated management of construction materials and components," Scientia Iranica, vol. 19, no. 3, pp. 381-392, 2012.

[31] Ł. Huminiecki, "Models of the gene must inform data-mining strategies in genomics," Entropy, vol. 22, no. 9, p. 942, 2020.

[32] B. Mohammed and A. M. Abdulazeez, "Journal of soft computing and data mining a review of principal component analysis algorithm for dimensionality reduction," Applied Soft Computing, vol. 2, no. 1, pp. 20-30, 2021.

[33] M. Yu, T. Quan, Q. Peng, X. Yu, and L. Liu, "A model-based collaborate filtering algorithm based on stacked AutoEncoder," Neural Computing \& Applications, vol. 6, no. 12, pp. 59-63, 2021.

[34] M. Shafiabadi, H. Pedram, M. Reshadi, and A. Reza, "An accurate model to predict the performance of graphical processors using data mining and regression theory," Computers \& Electrical Engineering, vol. 90, no. 1, p. 106965, 2021.

[35] N. Yusupova, G. Shakhmametova, and R. Zulkarneev, "Complex analysis of medical data with data mining usage," Acta Polytechnica Hungarica, vol. 17, no. 8, pp. 75-93, 2020.

[36] T. Van Nguyen, L. Zhou, A. Chong, B. Li, and X. Pu, "Predicting customer demand for remanufactured products: a data-mining approach," European Journal of Operational Research, vol. 281, no. 3, pp. 543-558, 2020.

[37] Y. L. Kang, L. L. Feng, and J. A. Zhang, "Research on subregional anomaly data mining based on naive Bayes," Computer Simulation, vol. 37, no. 10, pp. 303-306+316, 2020. 